\title{
Intercorrelation between MRI disease activity scores of the sacroiliac joints and the spine, and clinical disease activity indices in patients with axial spondyloarthritis
}

Reports in Medical Imaging

27 September 2017

Number of times this article has been viewed

Hon Wai Lau'

Chi Chiu Mok ${ }^{2}$

Wun Cheung Samuel Chan'

Ming Keung Yuen'

On Chee $\mathrm{Li}^{\prime}$

'Department of Radiology,

${ }^{2}$ Department of Medicine, Tuen Mun Hospital, Tuen Mun, New Territories, Hong Kong
Correspondence: Hon Wai Lau Department of Radiology, Tuen Mun Hospital, Tsing Chung Koon Road, Tuen Mun, New Territories, Hong Kong Tel +852 24685177

Email jackylauhw@gmail.com
Objective: To analyze the correlation between the magnetic resonance imaging (MRI) disease activity scores and the clinical disease activity indices (DAI) in Chinese patients with active axial spondyloarthritis ( $\mathrm{aSpA}$ ) that required biologics therapy. The correlation between MRI disease activity scores of the sacroiliac joints (SIJs) and the spine in these patients was also assessed.

Methods: This was a cross-sectional study design in which adult patients who fulfilled the Assessment of SpondyloArthritis international Society classification criteria for aSpA and had active disease (Bath Ankylosing Spondylitis Disease Activity Index score $\geq 4$, persistent spinal pain despite nonsteroidal anti-inflammatory drugs therapy $>3$ months) that required biologics therapy were included. The MRI disease activities were measured using the Spondyloarthritis Research Consortium of Canada (SPARCC) scores. Correlation between the SPARCC scores of the SIJs and the spine, and clinical DAI was calculated.

Results: Fifty-seven patients (47 men and 10 women; mean age $37 \pm 12$ years) completed the study. There was no statistically significant correlation between the SPARCC scores and clinical DAI. The SPARCC score of the SIJs showed a positive correlation with the SPARCC score of the spine $(r=0.34, p<0.05)$. The thoracic discovertebral units $(57 \%)$ were found to be more frequently associated with significant disease activity than the other levels of the spine.

Conclusion: MRI can detect active inflammation which may not be reflected by clinical DAI. MRI should be performed in patients with aSpA to provide a more comprehensive assessment and to better reflect disease activity and severity. MRI findings of more severe sacroiliitis should prompt the radiologist to look for involvement in the entire spine, particularly in the thoracic region.

Keywords: magnetic resonance imaging, MRI, SPARCC score, axial spondyloarthritis, ankylosing spondylitis, spondyloarthropathies, sacroiliitis

\section{Introduction}

Axial spondyloarthritis (aSpA) is a chronic inflammatory disease, predominantly affecting the sacroiliac joints (SIJs) and the spine in young adults. ${ }^{1}$ Various imaging modalities, including radiography, computed tomography (CT), and magnetic resonance imaging (MRI) can be used for assessment. MRI is the most sensitive for detecting early inflammatory changes in the SIJs and spine, and it is increasingly widely used for making diagnoses, monitoring disease activity, and guiding treatment of patients with aSpA. ${ }^{2-8}$ In fact, the Assessment of SpondyloArthritis International Society (ASAS) has included active inflammation in the SIJs on MRI as part of its latest classification 
criteria for aSpA, along with clinical spondyloarthropathy features (eg, inflammatory back pain) and blood tests (eg, HLA-B27).

Different scoring systems for MRI of the SIJs and the spine have been developed for more systematic and reliable assessment of active inflammation in patients with aSpA, such as Spondyloarthritis Research Consortium of Canada (SPARCC), ${ }^{9,10}$ Leeds, Aarhus, ${ }^{11}$ MR Imaging of Seronegative SpA, ${ }^{12}$ Ankylosing Spondylitis spine Magnetic Resonance Imaging-activity, ${ }^{13}$ and the Berlin methods. ${ }^{14,15}$ It has been reported that the SPARCC MRI index is the most reliable scoring system due to its high inter-reader reliability and sensitivity to changes. ${ }^{16-18}$

Despite the advantages of MRI, clinical disease activity indices (DAI), such as the Bath Ankylosing Spondylitis Disease Activity Index (BASDAI), ${ }^{19}$ the Assessment of Spondyloarthritis Disease Activity Score (ASDAS), ${ }^{20}$ the erythrocyte sedimentation rate (ESR), and C-reactive protein (CRP), are still more commonly used to assess the activity and severity of the disease in clinical practice due to ready availability. Nevertheless, various studies on biologics therapy for aSpA showed that the MRI disease activity scores may be the only indicator of disease response, ${ }^{5-8}$ implying that MRI disease activity scores may be more useful in guiding treatment plans compared to clinical DAI.

There is currently little published data on the correlation between the MRI disease activity scores and the clinical DAI, and that between MRI scores of the SIJs and the spine in Chinese patients with aSpA. ${ }^{21,22}$

The primary objective of this study was to investigate the correlation between the MRI disease activity scores and the clinical DAI in Chinese patients with active aSpA who required biologics therapy. In addition, the correlation between MRI disease activity scores of the SIJs and the spine in these patients was assessed.

\section{Methods}

\section{Study population}

This was a cross-sectional study in which patients were recruited from a regional hospital in Hong Kong in the period between June 2010 and March 2016. Inclusion criteria were adult patients older than 18 years of age, who fulfilled the classification criteria for aSpA as described by the ASAS, ${ }^{2}$ had active disease that required biologics therapy as defined by a BASDAI score $\geq 4$, and had persistent spinal pain despite nonsteroidal anti-inflammatory drugs therapy $>3$ months. Exclusion criteria were patients with a diagnosis of peripheral spondyloarthropathy without spinal involvement. The study was approved by the local institutional ethics committee (New Territories West Cluster Clinical \& Research Ethics Committee, Hospital Authority, Hong Kong). All patients provided written informed consent prior to participation in the study.

\section{Clinical assessments}

All patients included in the study were assessed in the rheumatology clinic. Blood tests were performed to determine the ESR and CRP level. Clinical disease activity was assessed using the ASDAS-ESR, the ASDAS-CRP, and the BASDAI. The patients' pain score and their global assessment were assessed using visual analog scale. Functional ability was assessed using the Bath Ankylosing Spondylitis Functional Index (BASFI) ${ }^{23}$ For the purpose of this study, the ASDASESR, ASDAS-CRP, ESR, CRP, BASDAI, patients' pain score, global assessment, and BASFI, were collectively designated as the clinical DAI.

For patients for whom anti-TNF treatment was planned or those switching from one to another, MRI was performed before the administration of the first dose of the new drug.

\section{MRI protocol}

It was subsequently arranged for patients to undergo MRI of the SIJs and whole spine. MRI examinations were performed by a 1.5 Tesla MRI scanner (Philips Achieva; Philips Healthcare, Best, the Netherlands). Spine and neurovascular coils for the SIJs, cervical, thoracic, and lumbosacral spine were used.

For the SIJs, the sequences were acquired in the coronal oblique plane parallel to its long axis ( $4 \mathrm{~mm}$ slice thickness; $0.4 \mathrm{~mm}$ gap; a total of 12 slices). The sequences included T1-weighted fast spin echo (FSE) (repetition time [TR]: 500-800 ms; echo time [TE]: 13-20 ms), and short tau inversion recovery (STIR) (TR: 2720-5000 ms; inversion time [TI]: $140 \mathrm{~ms}$; TE: $50 \mathrm{~ms}$ ).

The spine sequences were acquired in the sagittal plane ( $4 \mathrm{~mm}$ slice thickness; $0.4 \mathrm{~mm}$ gap; a total of 12 slices). Sequences included T1-weighted FSE (TR: 450-800 ms; TE: $7.4 \mathrm{~ms}$ ) and STIR (TR: 3000-5000 ms; TI: $150 \mathrm{~ms}$; TE: $80 \mathrm{~ms}$ ).

T1-weighted FSE sequences were used for anatomical reference while the STIR sequences were used for detecting acute inflammatory changes.

\section{MRI analysis}

Inflammation of the SIJs and spine was graded by the SPARCC scoring system, which is the most reliable scoring system with high inter-reader reliability and sensitivity to 
change. ${ }^{16-18}$ For scoring of the SIJs, each SIJ was divided into four quadrants. Increased signal intensity on STIR in each quadrant was scored. A total of six consecutive coronal oblique slices were assessed. An additional score of 1 was given if each SIJ on each slice had intense signal similar to that of pre-sacral blood vessels, or had a signal depth $>1 \mathrm{~cm}$. A patient's maximum SPARCC score of the SIJs would be 72 (Figure 1). For scoring the spine, each discovertebral unit was divided into four quadrants. Increased signal intensity on STIR in each quadrant was scored, and a total of three consecutive sagittal slices were assessed. Additional scores were given if each discovertebral unit on each slice had intense signal similar to that of cerebrospinal fluid, or had a signal depth $>1 \mathrm{~cm}$. The six discovertebral units with the highest scores were calculated, and a patient's maximum SPARCC score of the spine would be 108 (Figure 2).

Imaging analysis with quantification of the inflammation of the SIJs and spine was performed by two fellow musculoskeletal radiologists (with 2 and 10 years' experience in musculoskeletal imaging respectively) in consensus, who were blinded to the clinical DAI. The SPARCC scores of the SIJs and the spine, and the total SPARCC score (by adding up the two scores) were calculated. Distribution of the involved discovertebral units in the MRIs was also recorded. Image analysis was performed on a Picture Archiving and Communication System station (IMPAX; Agfa Healthcare N.V., Mortsel, Belgium).

\section{Statistical analysis}

Spearman's rank correlation coefficient was used to determine the correlation between the SPARCC scores and clinical DAI, as well as the correlation between the SPARCC scores of the SIJs and the spine. A $p$-value of $<0.05$ was considered statistically significant. Statistical analysis was performed using SPSS version 14.0 for Windows.

\section{Results}

Sixty patients were recruited for the study. Of the 60 patients, three patients were excluded due to incomplete MR examinations or incomplete clinical DAI. Fifty-seven patients completed the study, including 47 male and 10 female patients. Their mean age was $37 \pm 12$ years and their mean duration of disease was $5.5 \pm 5.2$ years.

\section{Descriptive statistics}

The mean ASDAS-ESR score was $4.1 \pm 0.9$. The mean ASDAS-CRP score was $3.7 \pm 0.9$. The mean BASDAI score was $6.0 \pm 1.8$.

Among the 57 MRI examinations, 48 (84.2\%) were positive for active inflammation based on the ASAS definitions for positive SIJ and spinal MRI.,4

The mean SPARCC score of the SIJs was $16.3 \pm 16.2$ (ranging from 0 to 64). The mean SPARCC score of the spine was $15.5 \pm 16.0$ (ranging from 0 to 54). Details of the clinical DAI and MRI disease activity scores are summarized in Table 1.
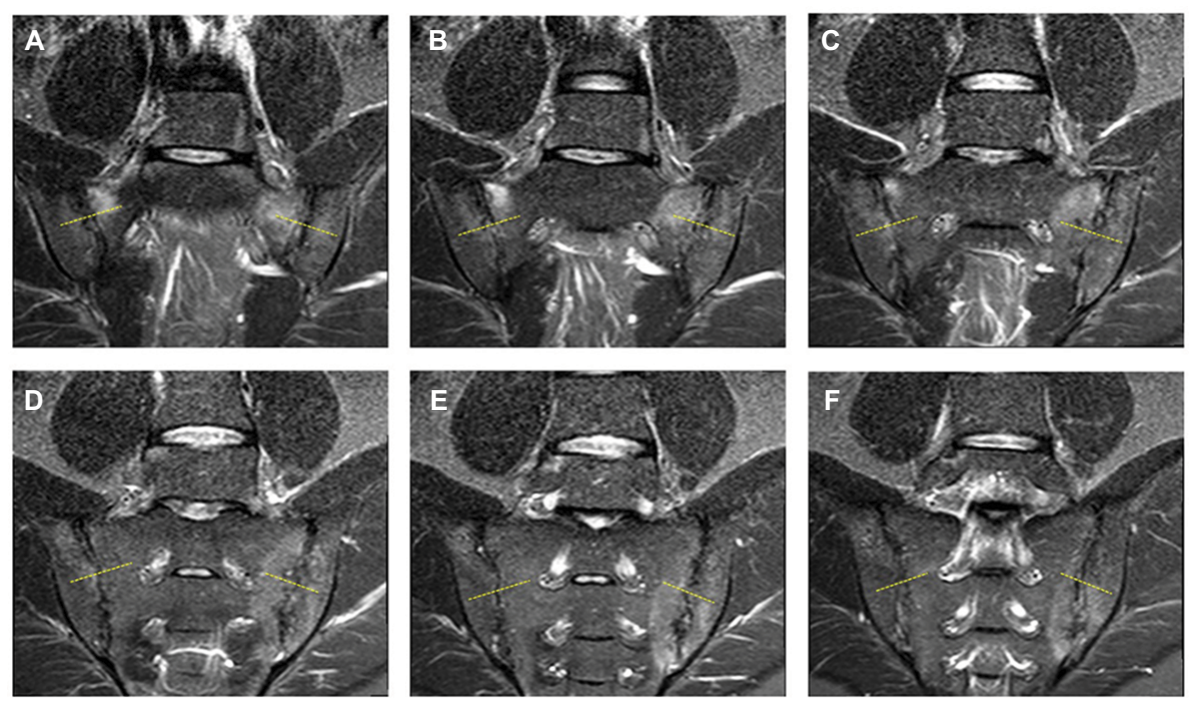

Figure I Six consecutive slices of coronal oblique STIR sequence of the MRI of SIJs (A-F) of a 39-year-old male patient with axial spondyloarthritis. Disease activity is scored using the Spondyloarthritis Research Consortium of Canada index. Each SIJ is divided into four quadrants (upper iliac, upper sacral, lower sacral, and lower iliac) by an imaginary horizontal dashed line and the SIJ line. Take the right SIJ of (B) as an example. The score is 4 (increased signal intensity in all four quadrants) + I (depth of signal $>\mathrm{I} \mathrm{cm}$ from articular surface) $+\mathrm{I}$ (intense signal similar to that of pre-sacral blood vessels) $=6$.

Abbreviations: MRI, magnetic resonance imaging; SIJs, sacroiliac joints; STIR, short tau inversion recovery. 

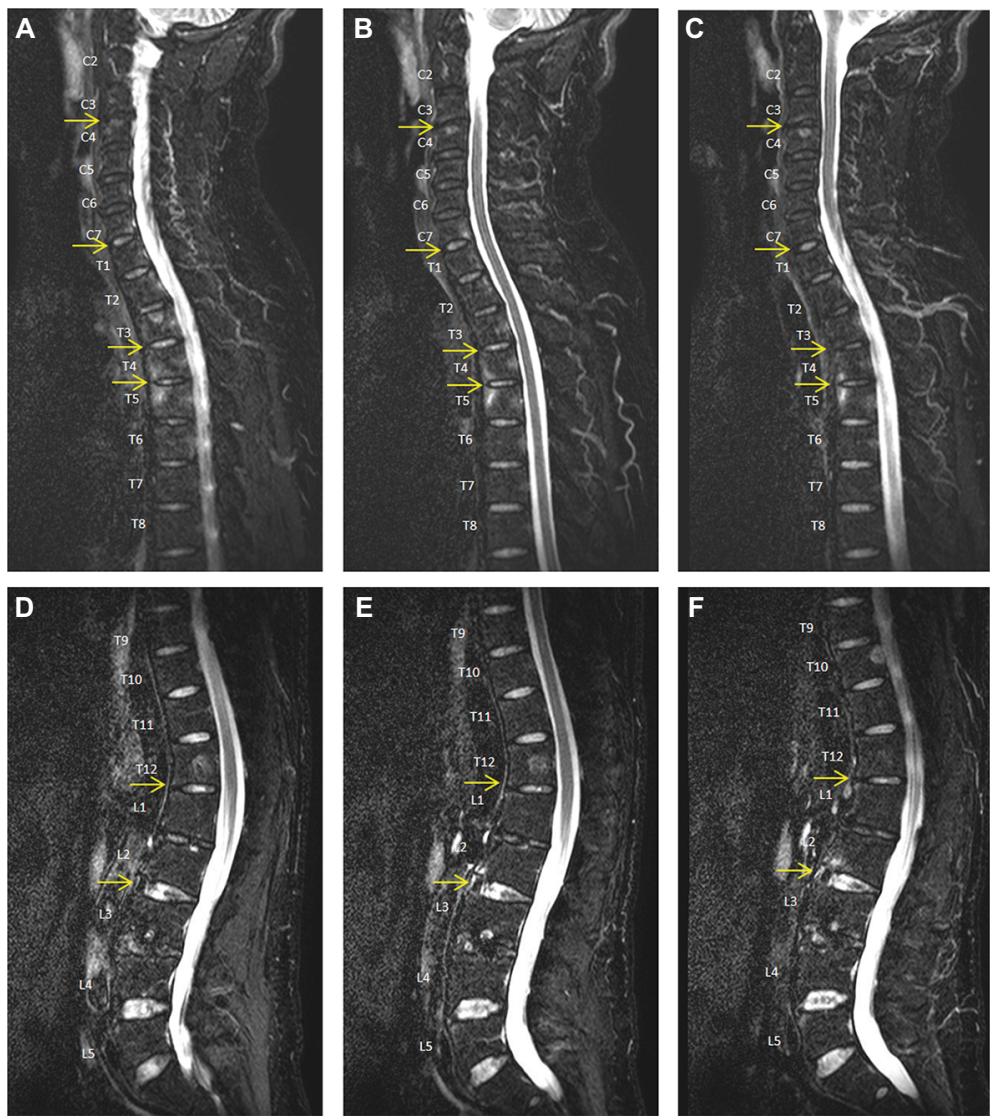

Figure 2 Three consecutive slices of sagittal STIR sequence of the MRI of cervicothoracic (A-C) and thoracolumbar (D-F) spine of a 45-year-old male patient with axial spondyloarthritis. Disease activity is scored using the Spondyloarthritis Research Consortium of Canada index. Each discovertebral unit is divided into four quadrants (anterosuperior, anteroinferior, posterosuperior, posteroinferior). Take the T4/5 level in (B) as an example. The score is 2 (increased signal intensity in anterosuperior and anteroinferior quadrants $+I$ (depth of signal $>I \mathrm{~cm})+I$ (intense signal similar to that of cerebrospinal fluid) $=4$. The six discovertebral units with the highest scores (arrows) are calculated.

Abbreviations: MRI, magnetic resonance imaging; STIR, short tau inversion recovery.

Table I MRI disease activity scores and clinical disease activity indices of the patients

\begin{tabular}{ll}
\hline Instruments or laboratory parameters & Mean \\
\hline SIJs SPARCC score & $16.3 \pm 16.2$ \\
Spine SPARCC score & $15.5 \pm 16.0$ \\
Total SPARCC score & $31.7 \pm 25.5$ \\
ASDAS-ESR & $4.1 \pm 0.9$ \\
ASDAS-CRP & $3.7 \pm 0.9$ \\
ESR & $45.8 \pm 29.6$ \\
CRP & $17.1 \pm 18.3$ \\
BASDAI & $6.0 \pm 1.8$ \\
Patients' global assessment & $6.4 \pm 2.1$ \\
Pain score & $6.6 \pm 1.9$ \\
BASFI & $4.8 \pm 2.4$ \\
\hline
\end{tabular}

Abbreviations: ASDAS, Assessment of Spondyloarthritis Disease Activity Score; BASDAI, Bath Ankylosing Spondylitis Disease Activity Index; BASFI, Bath Ankylosing Spondylitis Functional Index; CRP, C-reactive protein; ESR, erythrocyte sedimentation rate; MRI, magnetic resonance imaging; SIJs, sacroiliac joints; SPARCC, Spondyloarthritis Research Consortium of Canada.

A total of 200 discovertebral units were involved in the calculation of the SPARCC score of the spine in the study population. Twenty-three of these units $(11.5 \%)$ were in the cervical spine, $114(57 \%)$ were in the thoracic spine, and 63 $(31.5 \%)$ were in the lumbar spine. The thoracic discovertebral units were found to be more frequently associated with significant disease activity than the other levels of the spine.

\section{Correlation}

No statistically significant correlation between the SPARCC scores (SIJs, spine, and total) and clinical DAI including the ASDAS-ESR, ASDAS-CRP, ESR, CRP, BASDAI, the patients' global assessment, the patients' pain score, and BASFI was identified (Table 2).

The SPARCC score of the SIJs showed a statistically significant correlation with the SPARCC score of the spine $(r=0.34, p=0.009)$.

\section{Discussion}

Similar to previous studies in other populations, ${ }^{24-27}$ no statistically significant correlation was demonstrated between the SPARCC scores and any of the clinical DAI in Chinese 
Table 2 Correlation between the SPARCC scores and the clinical DAI using Spearman's rank correlation coefficient

\begin{tabular}{|c|c|c|c|c|c|c|}
\hline & \multicolumn{2}{|c|}{ SIJs SPARCC score } & \multicolumn{2}{|c|}{ Spine SPARCC score } & \multicolumn{2}{|c|}{ Total SPARCC score } \\
\hline & $\begin{array}{l}\text { Correlation } \\
\text { coefficient }(r)\end{array}$ & $p$-value & $\begin{array}{l}\text { Correlation } \\
\text { coefficient }(r)\end{array}$ & $p$-value & $\begin{array}{l}\text { Correlation } \\
\text { coefficient }(r)\end{array}$ & $p$-value \\
\hline SIJs SPARCC score & - & - & $0.344^{*}$ & 0.009 & $0.830 *$ & 0.000 \\
\hline Spine SPARCC score & $0.344^{*}$ & 0.009 & - & - & $0.767^{*}$ & 0.000 \\
\hline Total SPARCC Score & $0.830 *$ & 0.000 & $0.767^{*}$ & 0.000 & - & - \\
\hline ASDAS ESR & -0.013 & 0.925 & -0.010 & 0.943 & -0.020 & 0.885 \\
\hline ASDAS CRP & -0.086 & 0.524 & 0.014 & 0.915 & -0.064 & 0.636 \\
\hline ESR & 0.029 & 0.828 & 0.115 & 0.392 & 0.077 & 0.568 \\
\hline CRP & -0.136 & 0.314 & 0.112 & 0.405 & -0.040 & 0.767 \\
\hline BASDAI & -0.154 & 0.253 & -0.091 & 0.503 & -0.160 & 0.234 \\
\hline Patients' global assessment & -0.217 & 0.104 & -0.095 & 0.482 & -0.202 & 0.132 \\
\hline Pain score & -0.207 & 0.122 & -0.116 & 0.391 & -0.219 & 0.101 \\
\hline BASFI & -0.258 & 0.053 & -0.140 & 0.300 & -0.259 & 0.051 \\
\hline
\end{tabular}

Note: $* p<0.05$.

Abbreviations: ASDAS, Assessment of Spondyloarthritis Disease Activity Score; BASDAI, Bath Ankylosing Spondylitis Disease Activity Index; BASFI, Bath Ankylosing Spondylitis Functional Index; CRP, C-reactive protein; DAI, disease activity indices; ESR, erythrocyte sedimentation rate; SIJs, sacroiliac joints; SPARCC, Spondyloarthritis Research Consortium of Canada.

patients. For instance, MacKay et al, reported no statistically significant correlation between the SPARCC scores and the BASDAI, ASDAS-ESR, and ASDAS-CRP in a general aSpA outpatient population. ${ }^{27}$

A possible explanation for the lack of correlation is that some of the clinical DAI such as the BASDAI, patient's global assessment, pain score, and BASFI are subjective assessments dependent on individual patient experience which may be variable in terms of pain perception, sensitivity, and tolerance based on different demographics such as sex, age, and socioeconomic status. Large scale studies with subgroup analyses may be needed to further evaluate this aspect. In addition, pain due to inflammation in structures other than the SIJs and discovertebral units, such as capsulitis or facet joint arthritis, is not reflected in the commonly used MRI scoring systems, including the SPARCC scores which were used in this study. It may also be clinically difficult to locate pain originating from specific structures in the SIJs or spine. A more comprehensive MRI scoring system may need to be developed to eliminate this discrepancy, where issues such as long reporting time or inter-reader reliability are explored. Furthermore, patients may have developed symptoms unrelated to the acute inflammation which was the basis of most MRI scores. For example, patients with longstanding aSpA may develop mechanical pain from joint, muscle, or ligament related to joint fusion, deformity, or secondary degeneration, which would be more difficult to quantify and worth further evaluation.

Conversely, there were a few studies which reported a positive correlation between the SPARCC score of the SIJs and BASDAI score, ${ }^{21}$ and between the SPARCC score of the
SIJs and ASDAS score. ${ }^{22}$ Although the sample sizes of these studies were similar to the current study, the discrepancies in outcomes may be related to different patient baseline conditions such as disease severity. For instance, the patients in this study had a mean BASDAI score of 6.0, whereas those reported by Zhang et al had a mean BASDAI score of 3.6.2

Nevertheless, compared to clinical DAI, MRI can depict more objective features of active inflammation for early diagnosis and treatment monitoring. MRI scoring systems such as SPARCC have the additional benefit of offering a reproducible and reliable quantitative assessment of disease activity. Previous studies have demonstrated the ability of MRI disease activity scores to predict disease response after biologics therapy in patients with aSpA..$^{5-8}$ MRI disease activity scores are more likely to reflect short-term active inflammation, in which appropriate and prompt treatment can be given to prevent the development of irreversible structural changes which may cause long-term pain and disabilities, including joint deformity, secondary degeneration, and ankyloses. Therefore, MRI disease activity scores should be considered when deciding on disease management in addition to clinical DAI. Further development of a scoring system incorporating both MRI and clinical DAI scores may also lead to a more comprehensive assessment or better reflect disease activity and severity.

The study demonstrated a statistically significant correlation between the SPARCC score of the SIJs and the SPARCC score of the spine, an aspect that has not been widely discussed in previous studies. This finding illustrated that in patients with aSpA, the severity of SIJ inflammation has a positive correlation with that of spinal inflammation. 
Currently, the presence of sacroiliitis on imaging has been incorporated as one of the ASAS classification criteria for aSpA. However, there is conflicting evidence concerning whether it is beneficial to include inflammatory or structural lesions of spinal MRI as one of the classification criteria for diagnosing aSpA. A recent study by de Hooge et al. showed that $\geq 5$ inflammatory or fatty lesions seen on MRI of the spine were highly specific for aSpA, while some studies demonstrated that the use of MRI of the spine in addition to MRI of the SIJs had little incremental value for diagnosing patients with non-radiographic aSpA, with the concern of increasing false positive diagnosis. ${ }^{28-30}$ In our study, although the correlation between the SPARCC score of the SIJs and that of the spine is moderate $(r=0.34, p=0.009)$, the potential benefits of performing MRI of the spine in cases of aSpA in addition to MRI of the SIJs for supplementing useful information in diagnosis, monitoring of disease activity, and guiding treatment cannot be underestimated.

The thoracic discovertebral units of the spine (57\%) were the most frequently relevant to significant disease activity in the MRI, in accordance with findings reported in previous studies. ${ }^{31,32}$ A previous study by Lorenzin et al. suggested that the site of pain correlated with bone marrow edema in the thoracic spine, ${ }^{33}$ but not in the cervical or lumbar spine. Future studies could look into the feasibility of using scoring criteria on structural lesions (eg, fatty lesions) in thoracic spine for the diagnosis of aSpA. ${ }^{28}$ Nonetheless, the cervical (11.5\%) and lumbar discovertebral units (31.5\%) were also found to contribute to a substantial proportion of the significant disease activity. As a result, the entire spine should be examined as a complete assessment of active inflammation in a patient with aSpA.

There were several limitations to this study. Although the sample size is comparable to that of many other similar studies, it may not be sufficient to detect a significant correlation. In addition, the patients included were on the severe side of the disease spectrum, where the disease would be clinically more active and required biologics therapy, which was potentially a selection bias. Also, the time lapse between the clinical DAI evaluation and the MRI was mostly approximately 1-2 months. As a result, the pain or disability contributed by the active inflammatory component of the disease reflected in the clinical DAI may have already subsided when the patient underwent MRI, leading to a lower SPARCC score. On the other hand, a flare-up of the disease during the period of MRI may not have been assessed during the clinical evaluation.

\section{Conclusion}

In patients with aSpA, no significant correlation between the SPARCC scores and clinical DAI was demonstrated. However, as MRI can detect active inflammation which may not be reflected in the clinical DAI, it should be performed in aSpA patients to provide for a more comprehensive assessment of the disease activity and severity.

There was a statistically significant correlation between the SPARCC score of the SIJs and that of the spine. The spinal involvement was predominantly in the thoracic region. MRI findings of more severe sacroiliitis should prompt a radiologist to look for involvement in the entire spine, particularly in the thoracic region.

\section{Disclosure}

The authors report no conflicts of interest in this work.

\section{References}

1. Dean LE, Jones GT, MacDonald AG, Downham C, Sturrock RD, Macfarlane GJ. Global prevalence of ankylosing spondylitis. Rheumatology (Oxford). 2014;53(4):650-657.

2. Rudwaleit M, van der Heijde D, Landewe R, et al. The development of Assessment of SpondyloArthritis international Society classification criteria for axial spondyloarthritis (part II): validation and final selection. Ann Rheum Dis. 2009;68(6):777-783.

3. Rudwaleit M, Jurik AG, Hermann KG, et al. Defining active sacroiliitis on magnetic resonance imaging (MRI) for classification of axial spondyloarthritis: a consensual approach by the ASAS/OMERACT MRI group. Ann Rheum Dis. 2009;68(10):1520-1527.

4. Hermann KG, Baraliakos X, van der Heijde DM, et al. Descriptions of spinal MRI lesions and definition of a positive MRI of the spine in axial spondyloarthritis: a consensual approach by the ASAS/OMERACT MRI study group. Ann Rheum Dis. 2012;71(8):1278-1288.

5. Lambert RG, Salonen D, Rahman P, et al. Adalimumab significantly reduces both spinal and sacroiliac joint inflammation in patients with ankylosing spondylitis: a multicenter, randomized, doubleblind, placebo-controlled study. Arthritis Rheum. 2007;56(12): 4005-4014.

6. Gaspersic N, Sersa I, Jevtic V, Tomsic M, Praprotnik S. Monitoring ankylosing spondylitis therapy by dynamic contrast-enhanced and diffusion-weighted magnetic resonance imaging. Skeletal Radiol. 2008;37(2):123-131

7. Braun J, Baraliakos X, Hermann KG, et al. Golimumab reduces spinal inflammation in ankylosing spondylitis: MRI results of the randomised, placebo- controlled GO-RAISE study. Ann Rheum Dis. 2012;71(6):878-884.

8. Maksymowych WP, Dougados M, van der Heijde D, et al. Clinical and MRI responses to etanercept in early non-radiographic axial spondyloarthritis: 48-week results from the EMBARK study. Ann Rheum Dis. 2016;75(7):1328-1335.

9. Maksymowych WP, Inman RD, Salonen D, et al. Spondyloarthritis research Consortium of Canada magnetic resonance imaging index for assessment of sacroiliac joint inflammation in ankylosing spondylitis. Arthritis Rheum. 2005;53(5):703-709.

10. Maksymowych WP, Inman RD, Salonen D, et al. Spondyloarthritis Research Consortium of Canada magnetic resonance imaging index for assessment of spinal inflammation in ankylosing spondylitis. Arthritis Rheum. 2005;53(4):502-509. 
11. Puhakka KB, Jurik AG, Egund N, et al. Imaging of sacroiliitis in early seronegative spondylarthropathy. Assessment of abnormalities by MR in comparison with radiography and CT. Acta Radiol. 2003;44(2): 218-229.

12. Marzo-Ortega $\mathrm{H}$. Interreader agreement in the assessment of magnetic resonance Imaging of the sacroiliac joints in spondyloarthropathy-The 1st MISS study. Arthritis Rheum. 2002;46(Supp1):S428 [abstract].

13. Braun J, Baraliakos X, Golder W, et al. Magnetic resonance imaging examinations of the spine in patients with ankylosing spondylitis, before and after successful therapy with infliximab: evaluation of a new scoring system. Arthritis Rheum. 2003;48(4):1126-1136.

14. Hermann KG, Braun J, Fischer T, Reisshauer H, Bollow M. Magnetresonanztomographie der Sakroiliitis: Anatomie, Pathohistologie, MR-Morphologie und Graduierung [Magnetic resonance tomography of sacroiliitis: anatomy, histological pathology, MR-morphology, and grading]. Radiologe. 2004;44(3):217-228. German.

15. Rudwaleit M, Schwarzlose S, Listing J, Brandt J, Braun J, Sieper J. Is there a place for magnetic resonance imaging (MRI) in predicting a major clinical response (BASDAI 50\%) to TNF-alpha blockers in ankylosing spondylitis? Arthritis Rheum. 2004;50(9):S211.

16. Landewe RB, Hermann KG, van der Heijde DM, et al. Scoring sacroiliac joints by magnetic resonance imaging. A multiple-reader reliability experiment. J Rheumatol. 2005;32(10):2050-2055.

17. Lukas C, Braun J, van der Heijde D, et al. Scoring inflammatory activity of the spine by magnetic resonance imaging in ankylosing spondylitis: a multireader experiment. J Rheumatol. 2007;34(4):862-870.

18. van der Heijde D, Landewé R, Hermann KG, et al. Is there a preferred method for scoring activity of the spine by magnetic resonance imaging in ankylosing spondylitis? J Rheumatol. 2007;34(4):871-873.

19. Garrett S, Jenkinson T, Kennedy LG, Whitelock H, Gaisford P, Calin A. A new approach to defining disease status in ankylosing spondylitis: the Bath Ankylosing Spondylitis Disease Activity Index. J Rheumatol. 1994;21(12):2286-2291.

20. Lukas C, Landewe R, Sieper J, et al. Development of an ASAS-endorsed disease activity score (ASDAS) in patients with ankylosing spondylitis. Ann Rheum Dis. 2009;68(1):18-24.

21. Zhang P, Yu K, Guo R, Shah S, Morelli JN, Runge VA, Li X. Ankylosing spondylitis: correlations between clinical and MRI indices of sacroiliitis activity. Clin Radiol. 2015;70(1):62-66.

22. Cui Y, Zheng J, Zhang X, Zeng H, Luo R. Evaluation of treatments for sacroiliitis in spondyloarthropathy using the Spondyloarthritis Research Consortium Canada scoring system. Arthritis Res Ther. 2016;18(1):38.
23. Calin A, Garrett S, Whitelock H, Kennedy LG, O’Hea J, Mallorie P, Jenkinson T. A new approach to defining functional ability in ankylosing spondylitis: the development of the Bath Ankylosing Spondylitis Functional Index. J Rheumatol. 1994;21(12):2281-2285.

24. Goh L, Suresh P, Gafoor A, Hughes P, Hickling P. Disease activity in longstanding ankylosing spondylitis: a correlation of clinical and magnetic resonance imaging findings. Clin Rheumatol. 2008;27(4):449-455.

25. Aydin SZ, Bennett AE, McGonagle P, et al. Disease Activity Assessment by ASDAS Doesn't Predict Sacroiliac Inflammation with MRI in Axial SpA. Arthritis \& Rheumatism. 2010;62 Suppl 10:524.

26. van der Heijde D, Sieper J, Maksymowych WP, et al. Spinal inflammation in the absence of sacroiliac joint inflammation on magnetic resonance imaging in patients with active nonradiographic axial spondyloarthritis. Arthritis Rheumatol. 2014;66(3):667-673.

27. MacKay JW, Aboelmagd S, Gaffney JK. Correlation between clinical and MRI disease activity scores in axial spondyloarthritis. Clin Rheumatol. 2015;34(9):1633-1638.

28. de Hooge M, van den Berg R, Navarro-Compán V, et al. Patients with chronic back pain of short duration from the SPACE cohort: which MRI structural lesions in the sacroiliac joints and inflammatory and structural lesions in the spine are most specific for axial spondyloarthritis? Ann Rheum Dis. 2016;75(7):1308-1314.

29. Weber U, Zubler V, Zhao Z, et al. Does spinal MRI add incremental diagnostic value to MRI of the sacroiliac joints alone in patients with non-radiographic axial spondyloarthritis? Ann Rheum Dis. 2015;74(6):985-992.

30. Ez-Zaitouni Z, Bakker PA, van Lunteren M, et al. The yield of a positive MRI of the spine as imaging criterion in the ASAS classification criteria for axial spondyloarthritis: results from the SPACE and DESIR cohorts. Ann Rheum Dis. Epub 2017 Jun 29.

31. Maksymowych WP, Dhillon SS, Park R, Salonen D, Inman RD, Lambert RG. Validation of the spondyloarthritis research consortium of Canada magnetic resonance imaging spinal inflammation index: is it necessary to score the entire spine? Arthritis Rheum. 2007;57(3):501-507.

32. Baraliakos X, Landewe R, Hermann K, et al. Inflammation in ankylosing spondylitis: a systematic description of the extent and frequency of acute spinal changes using magnetic resonance imaging. Ann Rheum Dis. 2005;64(5):730-734.

33. Lorenzin M, Ortolan A, Frallonardo P, et al. Spine and sacroiliac joints on magnetic resonance imaging in patients with early axial spondyloarthritis: prevalence of lesions and association with clinical and disease activity indices from the Italian group of the SPACE study. Reumatismo. 2016;68(2):72-82.
Reports in Medical Imaging

\section{Publish your work in this journal}

Reports in Medical Imaging is an international, peer-reviewed, open access journal publishing original research, reports, reviews and commentaries on all areas of medical imaging. The manuscript management system is completely online and includes a very quick and fair peer-review system, which is all easy to use.

\section{Dovepress}

Visit http://www.dovepress.com/testimonials.php to read real quotes from published authors. 